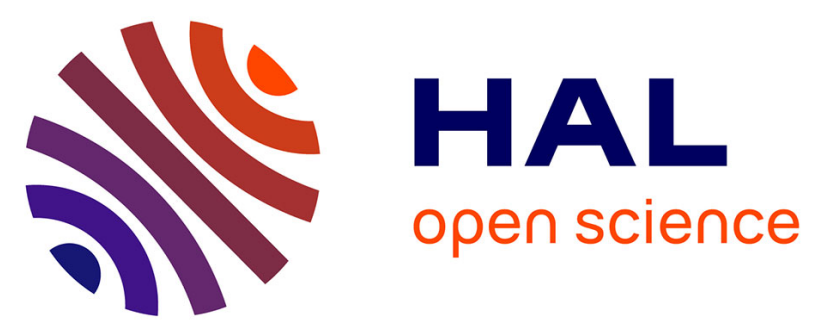

\title{
Telemedicine using mobile telecommunication: towards syntactic interoperability in teleexpertise
}

Mamadou Bilo Doumbouya, Bernard Kamsu-Foguem, Hugues Kenfack, Clovis

Foguem

\section{- To cite this version:}

Mamadou Bilo Doumbouya, Bernard Kamsu-Foguem, Hugues Kenfack, Clovis Foguem. Telemedicine using mobile telecommunication: towards syntactic interoperability in teleexpertise. Telematics and Informatics, 2014, vol. 31 (4), pp. 648-659. 10.1016/j.tele.2014.01.003 . hal-01005516

\section{HAL Id: hal-01005516 https://hal.science/hal-01005516}

Submitted on 12 Jun 2014

HAL is a multi-disciplinary open access archive for the deposit and dissemination of scientific research documents, whether they are published or not. The documents may come from teaching and research institutions in France or abroad, or from public or private research centers.
L'archive ouverte pluridisciplinaire $\mathbf{H A L}$, est destinée au dépôt et à la diffusion de documents scientifiques de niveau recherche, publiés ou non, émanant des établissements d'enseignement et de recherche français ou étrangers, des laboratoires publics ou privés. 


\section{Open Archive Toulouse Archive Ouverte (OATAO)}

OATAO is an open access repository that collects the work of Toulouse researchers and makes it freely available over the web where possible.

This is an author-deposited version published in: http://oatao.univ-toulouse.fr/ Eprints ID: 11456

To link to this article : DOI:10.1016/j.tele.2014.01.003

http://dx.doi.org/10.1016/j.tele.2014.01.003

\section{To cite this version:}

Doumbouya, Mamadou Bilo and Kamsu Foguem, Bernard and Kenfack, Hugues and Foguem, Clovis Telemedicine using mobile telecommunication: towards syntactic interoperability in teleexpertise. (2014) Telematics and Informatics, vol. 31 ( $\left.{ }^{\circ} 4\right)$. pp. 648-659. ISSN 0736-5853

Any correspondence concerning this service should be sent to the repository administrator: staff-oatao@listes-diff.inp-toulouse.fr 


\title{
Telemedicine using mobile telecommunication: Towards syntactic interoperability in teleexpertise
}

\author{
Mamadou Bilo Doumbouya ${ }^{\mathrm{a}, \mathrm{b}}$, Bernard Kamsu-Foguem ${ }^{\mathrm{b}, *}$, Hugues Kenfack ${ }^{\mathrm{a}}$, Clovis Foguem ${ }^{\mathrm{c}}$ \\ ${ }^{a}$ University of Toulouse, Faculty of Law, 2 rue du Doyen Gabriel Marty, 31042 Toulouse Cedex 9, France \\ ${ }^{\mathrm{b}}$ Laboratory of Production Engineering (LGP), EA 1905, ENIT-INPT University of Toulouse, 47 Avenue d'Azereix, BP 1629,65016 Tarbes Cedex, France \\ ${ }^{\mathrm{c}}$ Centre for Food and Taste Sciences (CSGA), UMR 6265 CNRS, UMR 1324 INRA, University of Bourgogne, 9 E Boulevard Jeanne d'Arc, 21000 Dijon, France
}

\section{Keywords:}

Telemedicine

Webservices

Mobile technologies

Interoperability
Telemedicine allows collaborative activities between health professionals for the deployment of medical procedures carried out remotely by means of device using information and communication technologies. This article focuses on the Teleexpertise that allows collaboration between medical professionals in order to share knowledge and expert advices used as explanation elements for decision support. We propose a conceptual model integrating the FIPA (Foundation for Intelligent Physical Agents) Contract Net Protocol which permits to collect medical professionals' answers for a request for teleexpertise in an efficient manner. Our model satisfies four requirements (coverage, QoS (Quality of Service) guarantees and prioritisation, mobility and roaming, service usability) on the configuration and operation of the underlying network and the services. Therefore, we provide an operational assistance by improvement of the networks quality of service via interoperable web services. Finally, we hope to bring a tangible contribution on the implementation of this suggested conceptualization that will allow to generate relevant and action-oriented findings.

\section{Introduction}

Telemedicine is the fact to use telecommunications and information technologies to provide health at a distance by gathering images and information for diagnosis, treatment, follow-up and monitoring.

Telemedicine is very helpful in several areas such as: rural area (Barjis et al., 2013) where medical expert advices are not very often available, critical area to provide quick health care.

Telemedicine is declined in five acts (DGOS, 2012) that are listed below:

- Teleconsultation: it allows a medical professional to consult a patient remotely.

- Teleexpertise: it allows a medical professional to seek remotely some opinions to his fellows who have the relevant training and skills. It can be achieved outside the presence of the patient.

- Telemonitoring: it allows a medical professional to monitor and supervise a patient remotely.

- Teleassistance: it allows a medical professional to assist remotely another medical professional during an intervention.

- Medical response: it is used to provide quick and efficient emergency services. The paramedics support the medical response of both air and ground ambulances.

\footnotetext{
* Corresponding author. Tel.: +33 6243023 37; fax: +33 562442708 .

E-mail addresses: mdoumbou@enit.fr (M.B. Doumbouya), bernard.kamsu-foguem@enit.fr (B. Kamsu-Foguem).
} 
Today with the rising of mobile devices many companies and research Institutes are trying to propose innovative solutions in the field of telemedicine adapted to mobile devices context. For example since 2009, the World Health Organisation (WHO) has been publishing reports covering initiatives and mobile health (Iwayaa et al., 2013). The mHealth Alliance (mHA), hosted by United Nations Foundation, is another institution who aims to maximise the impact of mobile health, especially in emerging economies, by ensuring interoperability and promoting open standards (Iwayaa et al., 2013). Actually, the mobile phone has been recognised as a possible tool of telemedicine since it became commercially available (Hung and Zhang, 2003). In this paper we follow this way i.e. proposing solutions including mobile devices related to the telemedicine.

In this study we will focus on the problem of telemedicine \& interoperability between many devices such as laptop, desktop, mobile devices, .

In the rest of this paper, we talk first about the objective we want to reach, second the state of art to show what has been already done, finally the others sections (Materials and methods, Results, Discussion) show how we try to reach our objective.

\section{Objective}

Nowadays the number of mobile devices is rising increasingly. These devices are being part of human being life because they are multi task, they can be used everywhere and every time and are more and more efficient.

By the definition of telemedicine, it allows the use of telecommunication and information systems and it permits collaboration between medical professionals. Telemedicine is value-adding, with the strength and responsiveness of practitioners supporting remote patients or other practitioners. Particularly, the teleexpertise is most likely to exhibit value added services through sharing of expertise, consensus-building and exchange of good practices. Thus our goal in this work is to bring innovative solutions to solve the problem of interoperability and to improve the networks quality of services on mobiles devices by using interoperable web services. Achieving this goal will permit to medical professionals to gain in efficiency.

\section{State of the art}

\subsection{Concept of interoperability}

Interoperability is neither the consistency nor the integration in one same system, even if it may however contribute. It concerns exchanges between computer applications, that these are located on the same computer or another player. For the European Committee for standardisation, interoperability is "a state between two applications when, for a specific task, an application can accept data from the other to perform this requires appropriate and satisfactory manner without this an external operator intervention" (Altran, 2010).

The main problem of Interoperability is the nature of exchanged data because these data come from multiples sources. The information circulating are more and more issued by the technical systems: sensors, cameras etc., and communications between human beings and the complexity of their relationships, they also pass by a series of systems, the keyboard on the phone through the cameras (Altran, 2010). The information systems have to handle these data regardless their sources.

According to Adebesin et al. (2013) there are four levels of interoperability:

- Technical level: It permits heterogeneous systems to exchange data, but it does not ensure that the receiving system with be able to use the exchanged data in a meaningful manner.

- Syntactic level: It ensures the preservation of the clinical purpose of the data during transmission among healthcare systems.

- Semantic level: It permits multiple systems to interpret the information that has been exchanged in a similar manner through pre-defined shared meaning of concepts.

- Organisational level: It makes easy the integration of business processes and workflows beyond the limits of a single organisation.

These levels are illustrated in Fig. 1.

In Adebesin et al. (2013), the authors talk about many standards around healthcare, and also make a mapping of these standards with the four levels of interoperability cited above. Messaging standards are generally aimed at supporting syntactic interoperability through the transmission of structured messages, while those classified under structure and content address interoperability both at the syntactic and semantic levels by specifying the structure of clinical documents that contain both coded and free text data. Clinical terminologies and codes are used to prevent ambiguity in the use of medical terms, thereby ensuring the same interpretation of clinical data, irrespective of the application that is receiving the data. For more details about the correspondence of interoperability levels and standards see Fig. 2.

\subsection{Solving interoperability issues}

In Altran (2010), the authors try to solve the interoperability problem layer by layer. They give in each layer which technologies can be used. They divided their illustration into nine layers that are listed below: 


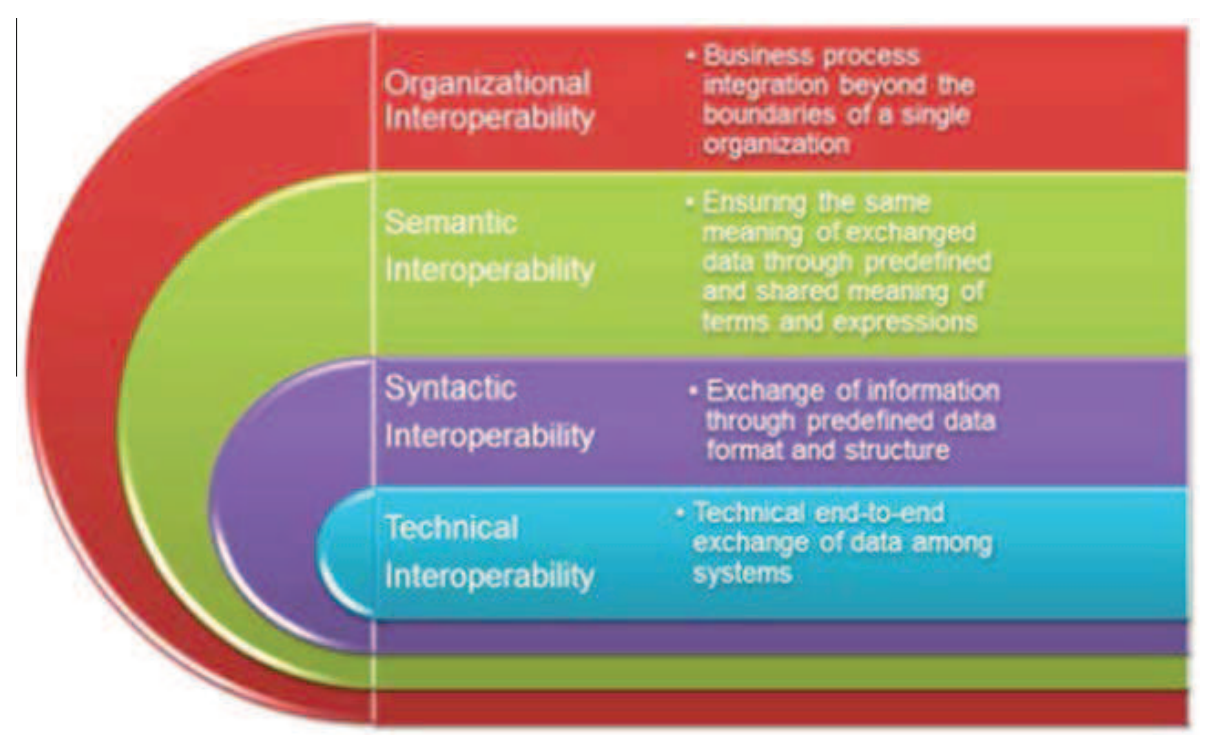

Fig. 1. Levels of interoperability (Adebesin et al., 2013).

\begin{tabular}{|c|c|c|c|c|}
\hline \multirow[b]{2}{*}{ Standard } & \multicolumn{4}{|c|}{ Interoperability level } \\
\hline & $\frac{\bar{E}}{\underline{E}}$ & 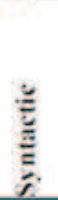 & 兽 & 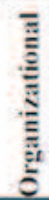 \\
\hline \multicolumn{5}{|c|}{ Identifiers } \\
\hline ISO TS $22220: 2011$ & & $\mathrm{X}$ & $\mathrm{X}$ & \\
\hline ISO TS $27527: 2010$ & & $\mathrm{X}$ & $\mathrm{X}$ & $\mathrm{X}$ \\
\hline \multicolumn{5}{|c|}{ Messaging / information exchange } \\
\hline HL7 V2X & & $\mathrm{X}$ & $\mathrm{X}$ & \\
\hline HL7 V3 & & $\mathrm{X}$ & $\mathrm{X}$ & \\
\hline DICOM & & $\mathrm{X}$ & $\mathrm{X}$ & \\
\hline SDMX-HD & & $\mathrm{X}$ & & \\
\hline \multicolumn{5}{|c|}{ Structure and content } \\
\hline ASTME2369-12 & & $\mathrm{X}$ & $\mathrm{X}$ & \\
\hline HL7CDA & & $\mathrm{X}$ & $\mathrm{X}$ & \\
\hline HL $7 / A S T M C C D$ & & $\mathrm{X}$ & $\mathrm{X}$ & \\
\hline HL7 CRS & & $\mathrm{X}$ & $\mathrm{X}$ & \\
\hline ISO 21090 & & $\mathrm{X}$ & $\mathrm{X}$ & \\
\hline \multicolumn{5}{|c|}{ Clinical terminology and coding } \\
\hline & & & & \\
\hline SNOMED & & $\mathrm{X}$ & $\mathrm{X}$ & \\
\hline LOINC & & $\mathrm{X}$ & $\mathrm{X}$ & \\
\hline ICD & & $\mathrm{X}$ & $\mathrm{X}$ & \\
\hline $1 C P C-2$ & & $\mathrm{X}$ & $\mathrm{X}$ & \\
\hline CPT & & $\mathrm{X}$ & $\mathrm{X}$ & \\
\hline \multicolumn{5}{|c|}{ Electronic health record } \\
\hline ISO $18308: 2011$ & & $\mathrm{X}$ & $\mathrm{X}$ & $\mathrm{X}$ \\
\hline \multicolumn{5}{|c|}{ System functional models } \\
\hline $\begin{array}{l}\text { HL } 7 \text { EHR-System Functional Model. } \\
\text { Release } 1.1\end{array}$ & $\mathrm{X}$ & $\mathrm{X}$ & $\mathrm{X}$ & $\mathrm{X}$ \\
\hline \multicolumn{5}{|c|}{ Security and access control } \\
\hline ISO TS 22600 & & & & $\mathrm{X}$ \\
\hline
\end{tabular}

Fig. 2. Standards and interoperability level mappings (Adebesin et al., 2013).

- Physical level, network: This level is never specific to health. It unifies around Internet and mobile technologies but can evolve, as demonstrated by the developments in the mobile. Finally, the business needs (for a new service, for example) must be treated as independently as possible.

- Transport and communication protocols: At the international level, coordination occurs in EDI (Electronic Data Interchange) structures which have been introduced and established between professionals and regulatory Web sectors (UN/CEFACT, ebXML).

- Process and business system exchange: This level is supported in health by the international organisation called IHE (Integrating the Healthcare Enterprise) whose role is to define "integration profiles" for different processes. 
- Documents and messages structure: This level is defined by standards such as HL7 (Health Level 7), DICOM (Digital Image Communication). There is also IHE which defines integrations profiles between HL7 and DICOM systems.

- Definition and information structure: Whether it is an observation or a medical Act, the mandatory elements are included in a manner that is scientifically sound and ethically acceptable.

- Semantics: An effort is being revived on the integration of semantics terminologies in information systems. Examples of universal standard terminologies are: SNOMED (Systematised Nomenclature of Medicine) and for indexation, storage, retrieval, and aggregation of medical data across specialties; LOINC (Logical Observation Identifiers Names and Codes) for identifying medical laboratory observations.

- Ergonomics: Its importance is often underestimated. The standards for presentation and disclosure of information will be required for all cases where a human actor will be involved in activities actively or passively.

- Security: It is a global approach and must be the subject of an independent reflection, a protection of personal data and safe interaction with the technologies. We must ensure an equivalent level of protection of patients' personal data in line with international standards on fundamental rights, privacy and the legitimate interest of legal persons.

- Computer languages: They facilitate the work on information management mechanisms and their dependability, dynamic update processes, advanced procedures and formalised principles for interoperability to support organisational and functional purposes in dynamic and mobile environments.

These different layers are illustrated in Fig. 3.

Unlike the previous paragraph, European Community organises the implementation of standards for interoperability according to three levels (DGOS, 2012) instead of nine in Altran (2010):

- The high level: This level concerns the specifications defined by the national and territorial projects;

- The intermediate level: It concerns the profiles of integration to meet the main challenges;

- The low level: It concerns the standards (HL7, DICOM, IEEE, W3C, Oasis, ...), interfaces between systems.

\subsection{Solving interoperability problems by FIPA Contract Net Protocol}

The Foundation for Intelligent Physical Agents (FIPA) is an international organisation that dedicated to promoting the industry of intelligent agents by openly developing specifications supporting interoperability among agents and agent-based applications. This occurs through open collaboration among its member organisations, which are companies and universities that are active in the field of agents. FIPA makes the results of its activities available to all interested parties and intends to contribute its results to the appropriate formal standards bodies (FIPA).

The FIPA Contract Net Protocol is an interaction protocol for task allocation (Liekna et al., 2012) respecting FIPA specifications. It is designed for distributing one task among a number of agents(Knabe et al., 2002). In this protocol there are two types of agents:

Ergonomics

\section{Semantics}

SNOMED, LOINC, NGAP, CCAM

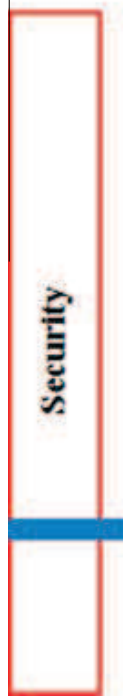

Definition and Information structure

Data representation, EHRCOM archetype

Documents and messages

Models, messages structures, and documents (CDA) - HL -

Process and business system exchange (medical, ass-

desease...)-Integration Profiles, envelopes... IHE

Transport and Communication protocols

HTTP, SMTP, professionals extensions, Webservices

\section{Network}

Physical media, protocols, management - several possibilities and IP

Network layer or others

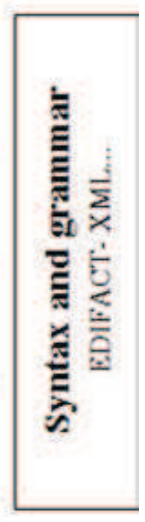

Fig. 3. Independant layers (Altran, 2010). 
- Initiator: The manager agent which has some tasks to perform.

- Participants: The other agents which participate in ongoing and future planned tasks.

At any time, any agent can be an Initiator, Participant or both. The flow of the protocol is the following (See figure below for the UML ${ }^{1}$ protocol diagram.) The initiator asks for proposals from other agents by issuing a Call for Proposals (CFP) that specifies the tasks and any additional conditions for its execution. Participants receiving the CFP are potential contractors and can submit their proposals to perform task that includes preconditions of the execution of the task like price, time, and technical performance when the task will be done or any other precondition. Alternatively, agents may refuse to answer to a CFP where it considers the request unnecessary in the circumstances. As soon as the deadline passes, the initiator evaluates the received proposals and selects agents to perform the task. It can choose one, several or none if there are no satisfactory proposals. The agent (s) of the selected proposal (s) will be sent acceptance notification, and the remaining agents will receive rejections. As soon as the initiator accepts the proposal, the participant has a commitment to carry out the task, i.e. the proposal is binding to the participant. After completing the task the participant sends the completion message to the initiator that can be either information that the task is done or a recorded description of the results contained in the completed task report. In case of failure a corresponding message is sent. Additionally, not-understood messages can be sent at any moment of the protocol in case the agent did not understand the previous message (FIPA; Liekna et al., 2012).

Concerning the goal we want to reach, this protocol is not very suitable for collaboration between medical professionals because as it said in Liekna et al. (2012): "in general the Contract NET protocol allows finding the most suitable agent for a single task by comparing proposals submitted by agents. Still it is not clear how to use it optimally for multiple tasks at the same time". We want to collect the proposals of all medical professionals participating in the collaboration. But we can reuse the principle used in FIPA Contract Net protocol to establish communication between agents and also the principle used to collect the agents' proposals. Fig. 4 illustrates how the FIPA Contract Net protocol operates.

\subsection{Mobile telemedicine}

Mobile telemedicine is the fact of using wireless communication services to deliver a medical service (Batistatos et al., 2012).

Many works have been achieved concerning this field, for example in Batistatos et al. (2012) the authors proposed a solution of mobile telemedicine in the context of moving vehicle (ambulance). In Kulkarni and Ozturk (2011), the authors proposed a solution called mPHASIS. This solution is designed to be pervasive and ubiquitous (Kulkarni and Ozturk, 2011). It is also a complementary solution for the hospital information system in the sense that it can be coupled to hospital information system by the use of webservices (Kulkarni and Ozturk, 2011). In Lai et al. (2009), it is proposed a mobile telemedicine solution based on wireless multi-hop. This solution permits to provide home care monitoring applications for elderly persons.

\section{Materials and methods}

Our contribution in this work will focus on Teleexpertise i.e. proposing collaborative solutions for medical professionals using information systems supporting mobile technologies. Globally we want to propose solutions to solve the interoperability problem encountered in Teleexpertise act through the use of several devices such as mobile devices. The main actors collaborating in this telemedicine act are essentially medical professionals.In fact a medical professional called Requesting physician requests a remote medical professional called Required physician to seek advice or to share some information about patients. These solutions will permit to medical professionals to gain in efficiency because they could be reached anywhere and everywhere if they are equipped with mobile devices. Fig. 5 below shows a generic illustration of the act of teleexpertise:

In teleexpertise the medical professional sometimes had to seek advice about very profound issues in particularly problematic circumstances and under some time constraints. As said in DGOS (2012), the data sharing feature follows two implementation models:

- Model of collaboration with strong coupling:

- Participants have a shared vision (in a paired exchange) of the same document from a main workstation.

- Participants have an identical vision of a same document previously distributed locally on each workstation. The distributed application on each workstation provides a shared vision of this document by ensuring an identical simultaneous presentation.

- Model of collaboration with weak coupling:

- Participants use the same application (Software as a service (SaaS) mode or Following pre-registration in an integral part of cloud-computing solutions) to access the same information and documents without a common view and the

${ }^{1}$ Unified Modeling Language. 


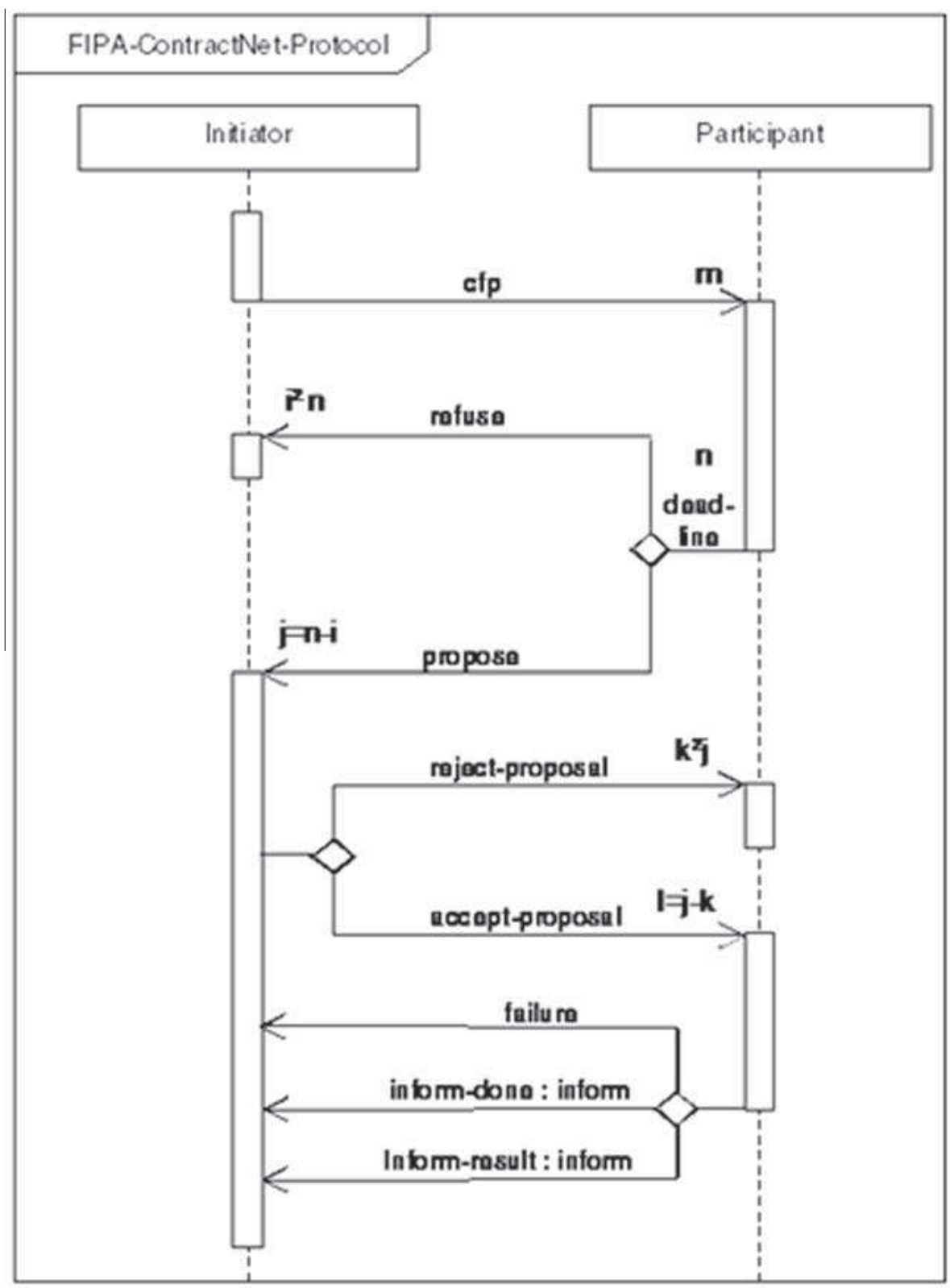

Fig. 4. FIPA Contract Net Protocol.

joint planning process (each actor can evolve in the document and be at the screen on a different page of the other actors involved in the Act).

French Telemedicine Committee identified five national priorities (DGOS, 2012) of telemedicine deployment, so our study will focus on these priorities. These five priorities are:

- Permanence of care in medical imaging

- Management of care for stroke

- Health of inmates

- Management of chronic diseases

- Care in medico-social structures

4.1. Communicating medical devices

According to DGOS (2012), there are two main types of devices which can be used in medical communication:

- Active implantable medical devices such as implantable cardioverter-defibrillators. 


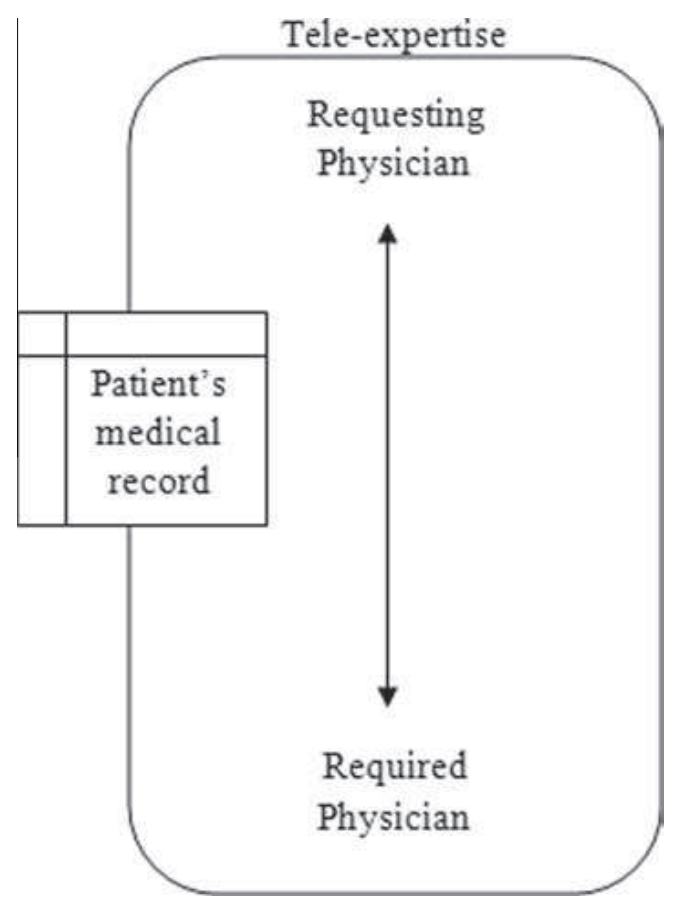

Fig. 5. Teleexpertise act in telemedicine.

- Non-implantable medical devices namely those with manual entry: touch screen terminal set up at the patient's home or remote access via web interface (Pc, Tablet, smartphone,...) and components that are suitable for both mobile and fixed applications, including sensors and biomedical devices.

\subsection{Our proposed model}

In DGOS (2012), the authors give some generic specifications related to these five priorities cited above. Our proposition will related to the health of inmates. We propose an UML use case diagram that shows some main purposes of collaboration between medical professionals. Fig. 6 shows an illustration of our model. Our proposition will focus on the physical level and the transport and communication protocols level cited above, in other words technical interoperability.

We suppose that the data concerning a patient is already available on a server.

In this model, the actors can use both mobile devices and PC. For example the requesting physician can use a mobile device such a tablet or a PC to access a patient medical record. Also via an application he can request for an expertise. This requests is broadcasted to all the specified required physicians by a webserver. The required physicians can be notified of this request directly on their mobile device. So they can then intervene in the act of teleexpertise wherever they located if they accept the request. This step can be achieved by the principle used in FIPA Contract Net Protocol to contact the different participants. We will call this principle of FIPA Contract Net Protocol mFCNP for modified FIPA Contract Net Protocol.In this $m F C N P$ we replace propose by accept which means that the corresponding medical professional accepts to participate in the current expertise act. This principal is illustrated in Fig. 7.

Here in our model the initiator is the server and the participants are the required physicians and the deadline specifies when the expertise act must be done. The communication purpose can be done via visioconference and also by sharing documents. Thus when the visioconference is chosen, the network layer can be dimensioned to support the visioconference according to the required quality of service. For more details about the required quality of service you can see DGOS (2012). In Fig. 8, we illustrate the communication between the actors in the act of teleexpertise by UML (uml) sequence diagrams.

Generally, this diagram shows the establishment of the communication between medical professionals. The intermediate device (A or B) are the devices used by the physicians in the act of teleexpetise. These devices can be smartphone, PC, or laptop. The ref $m F C N P$ represents our modified Fipa Contract Protocol sequence diagram above. It permits to collect the answer of the physicians for the current request for expertise. Ref teleepertise sequence diagram 2 (Fig. 9) illustrates the data exchange practices, the collection of medical professionals' advices and the creation of report that will be used by the requesting physician to provide treatment according to consensual and reliable bases with the patient's consent.

Concerning the transport layer, we propose to use web services for data exchange. And the format of data exchanged is $X M L(x \mathrm{ml}),{ }^{2}$ because it has becoming an important standard in computer science, it was utilised as an import-export format in web services or other collaborative platforms (Figueredo and Dias, 2004). Therefore the designed architecture should be Service

\footnotetext{
2 Extensible Markup Language.
} 


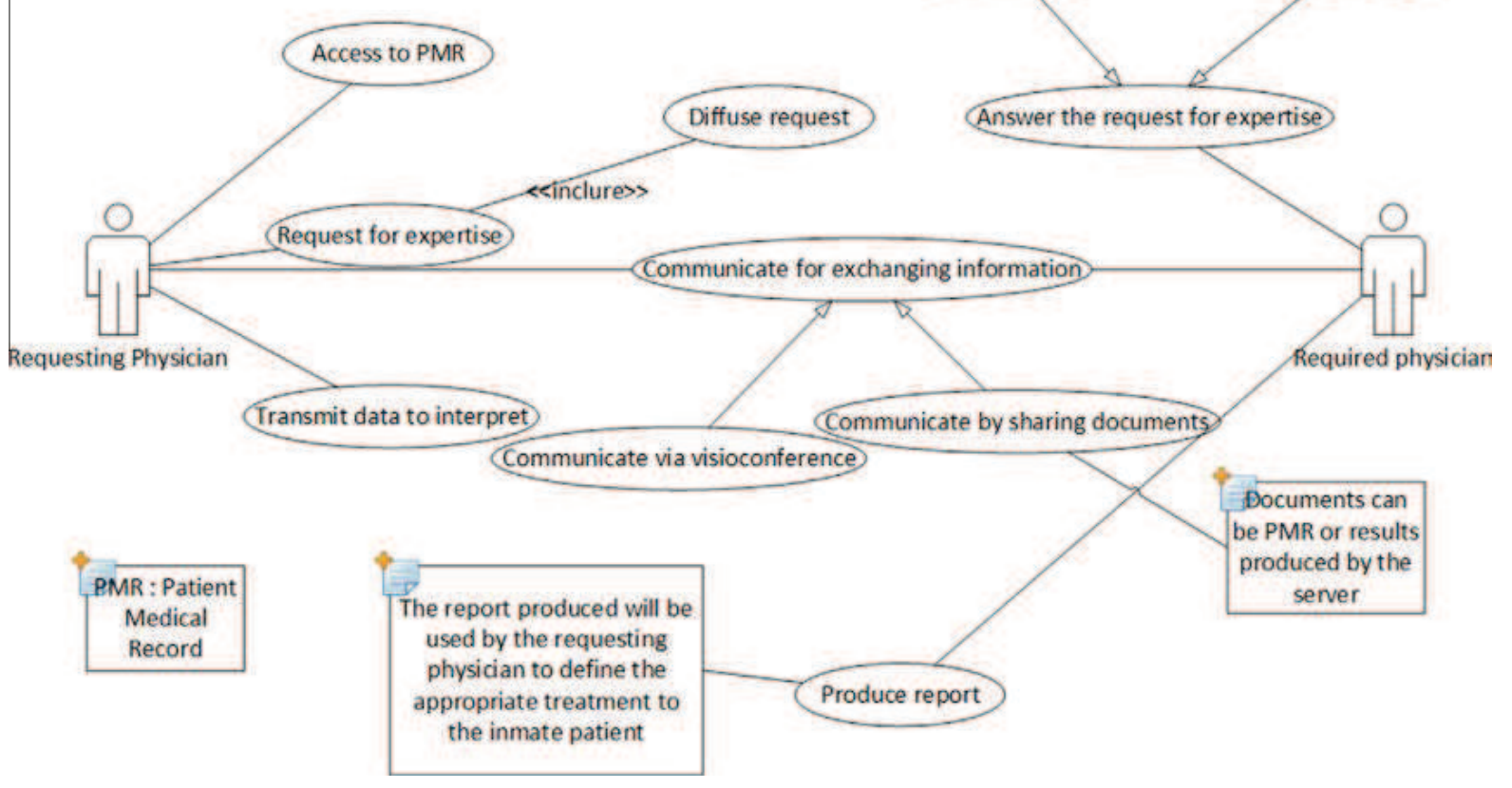

Fig. 6. Teleexpertise use cases.

Oriented Architecture (SOA) using the SOAP (Simple Object Access Protocol) for exchanging structured information and communication between devices. The architecture of the proposed model is depicted in Fig. 10.

\section{Results}

The originality of our work is the fact that we integrate in our model a modified version of Fipa Contract Net Protocol. This protocol was originally introduced to guarantee a reliable collaboration between different participants which trying to

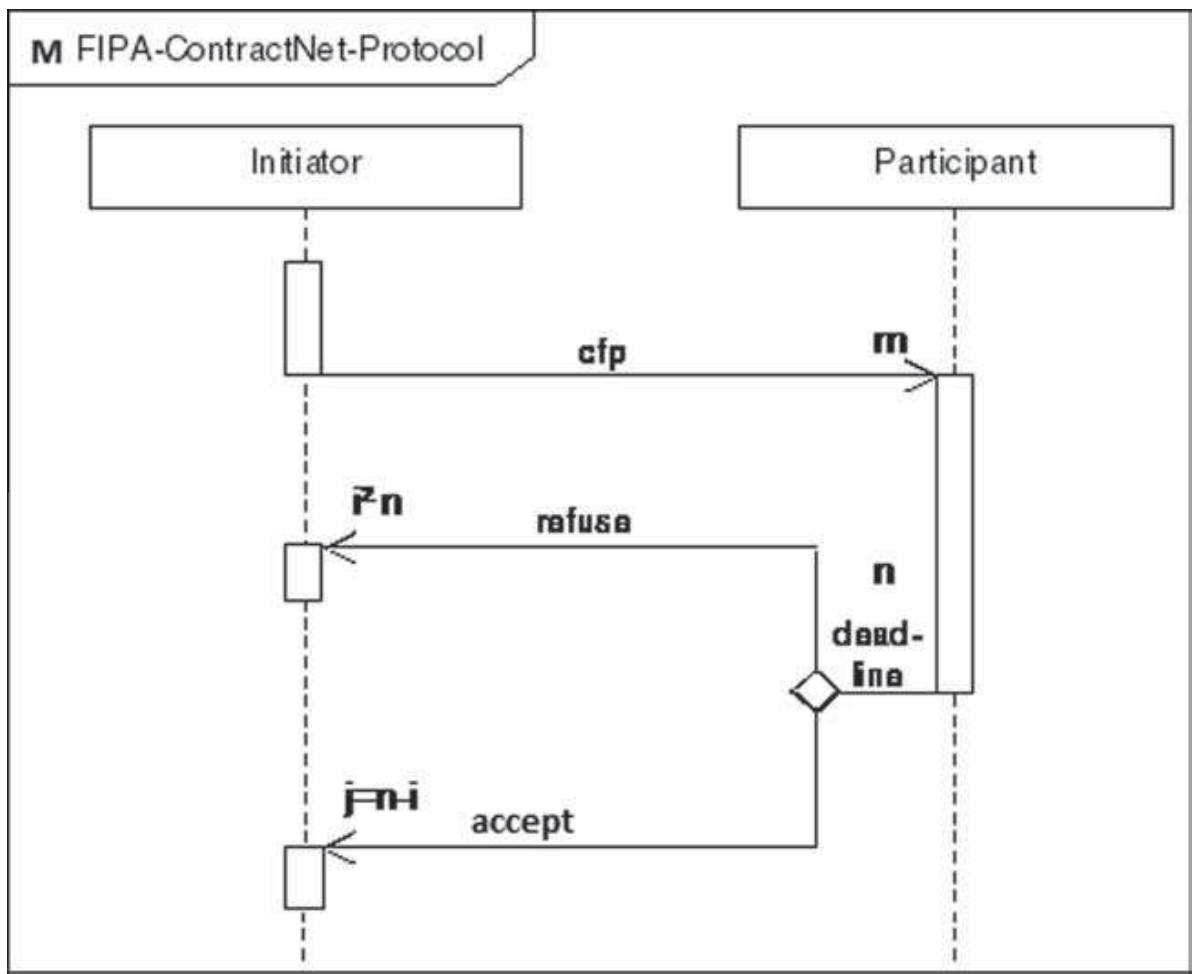

Fig. 7. M-FIPA Contract Net Protocol. 


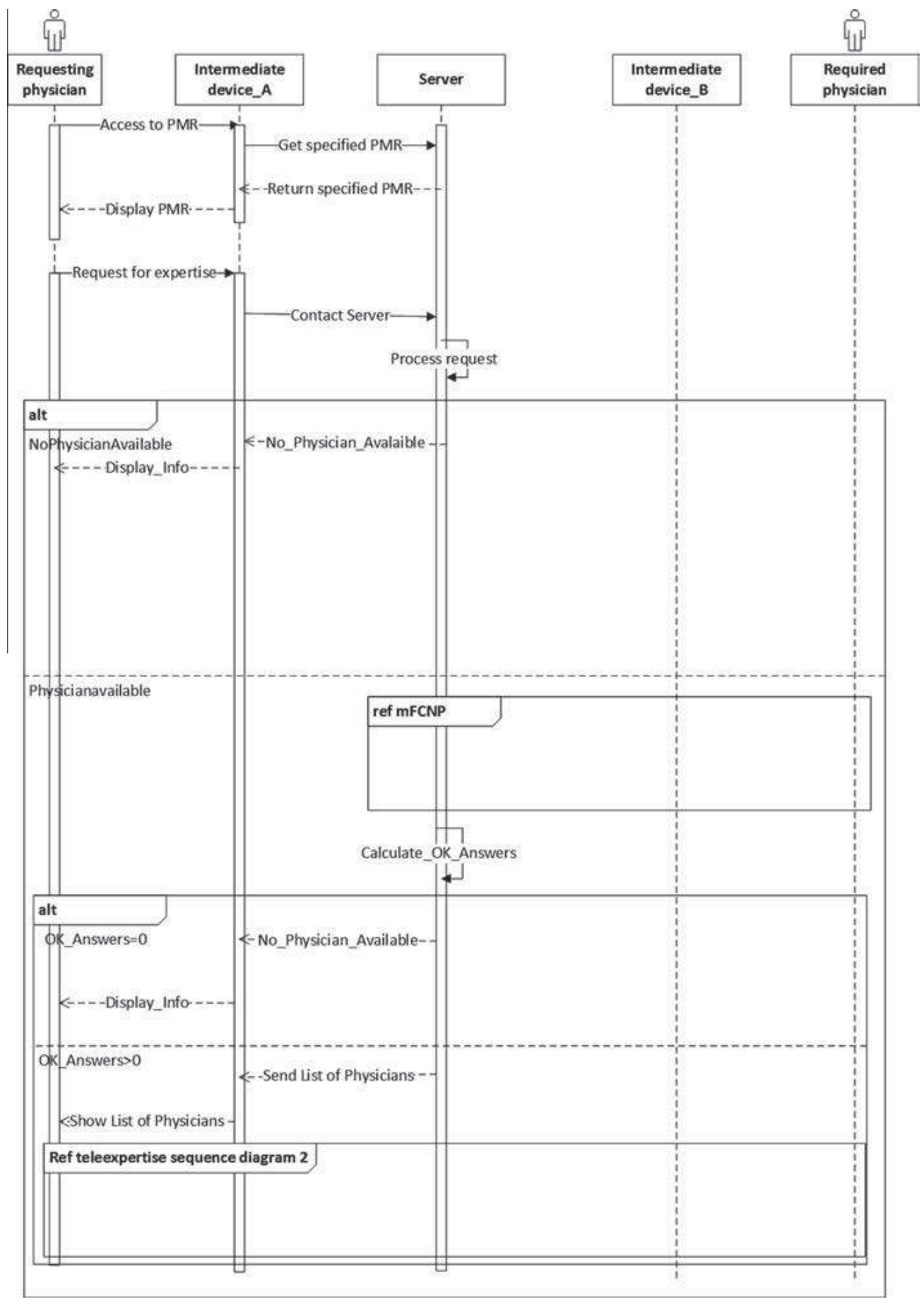

Fig. 8. Teleexpertise sequence diagram 1.

achieve the same goal. It allows to collect participants' answers and in the same occasion it identifies the participant which don't reply to the request. Since this protocol includes a deadline time, this time will specify the urgency of the action plan for needed services. It is fundamental for the quality of interaction that promotes the implementation of telemedicine activities with following the levels of priority listed in the contextualised plan.

The emergency telemedicine applications and their usage scenarios impose a number of requirements on the configuration and operation of the underlying network and the services it provides (e.g., data services) Mutafungwa et al., 2012.

Our model satisfies four of these requirements namely:

- Coverage: The mains actors are equipped with mobile devices, so they can act wherever and whenever they are located. This guarantees the coverage requirement.

- QoS guarantees and prioritisation: We advice to follow the QoS specifications gave in DGOS (2012). So that the proposed model will meet the required QoS for telemedicine applications. 


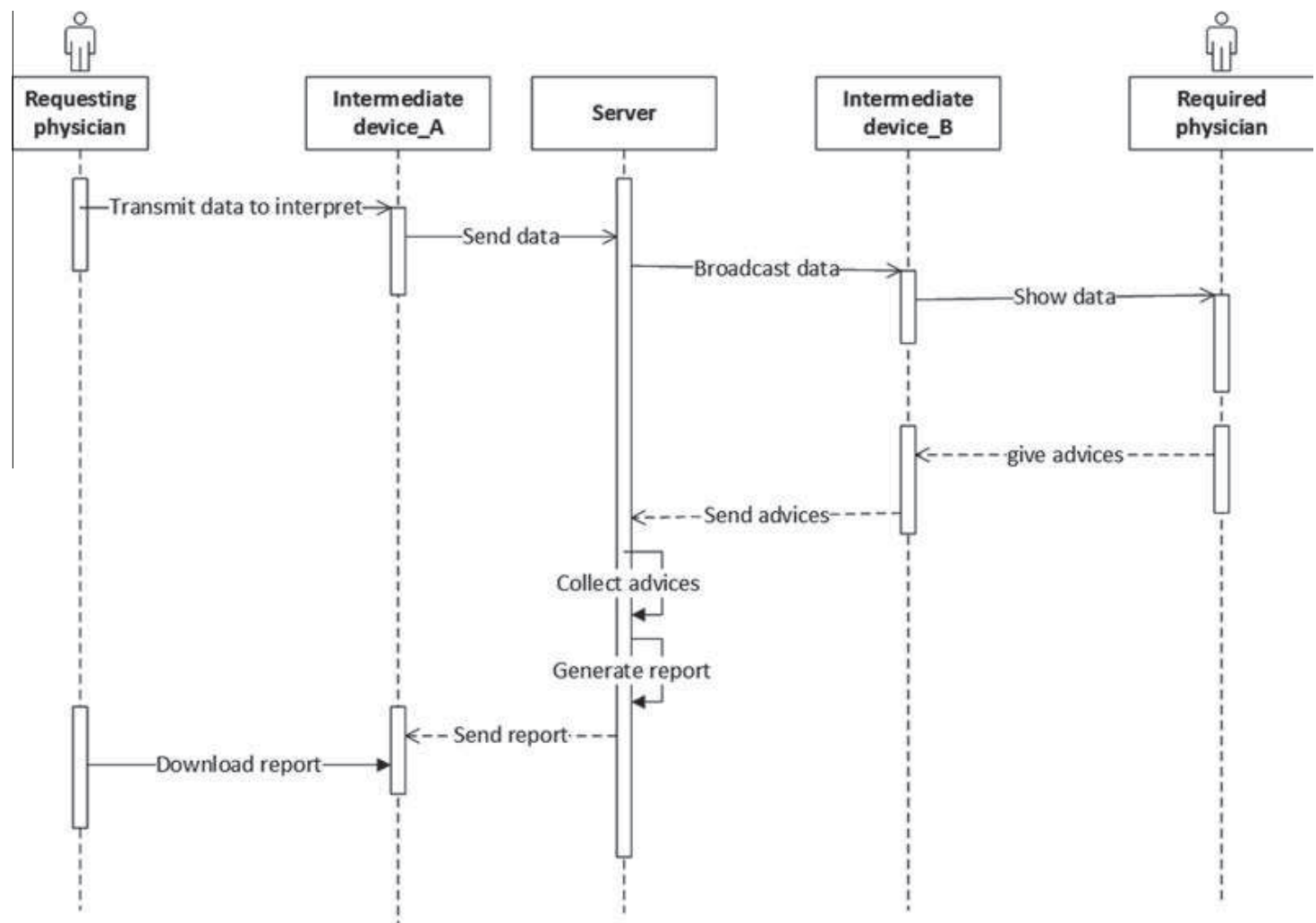

Fig. 9. Teleexpertise sequence diagram 2 .

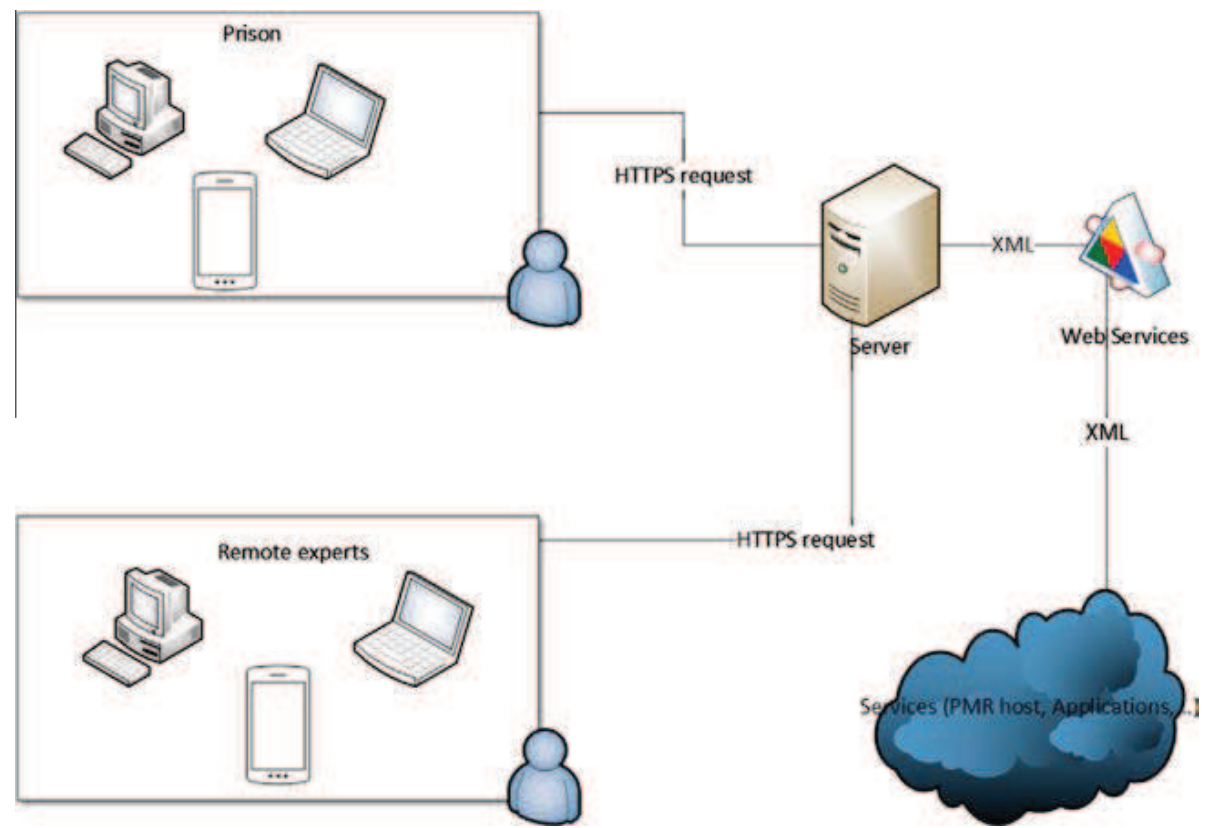

Fig. 10. Teleexpertise architecture.

- Mobility and roaming: This requirement is guaranteed by mobility property of the mobile devices.

- Service usability: Our model reaches this requirement because we propose that the network layer can be dimensioned automatically according to the required QoS when a communication mode (visioconference or sharing document) is chosen.

The proposed system is not yet implemented. However with the used technologies such as SOAP relied on XML Information Set specifying message formats, it assumed to solve the syntactic interoperability issues of medical professionals acting for the inmates health. And also by the use of HTTPS ${ }^{3}$ protocol we guarantee a secure transportation of the data exchanged.

\footnotetext{
${ }^{3}$ HyperText Transfer Protocol Secure.
} 
As a result, with this solution the actors can use both mobile devices and PC to communicate the core information of a critical care management and to innovate with these new possibilities.

\section{Discussion}

In DGOS (2012) and Altran (2010), the authors gave some generic specifications of the implementation of telemedicine acts. In our work we proposed a solution of teleexpertise for inmate health and show that this guarantees interoperability (message negotiation and transmission) between several types of devices by the use of SOAP protocol.

In the proposed model, the participants can achieve the act of teleexpertise by using visioconference, sharing documents or both can be used during any activity. So particularly for the mobile devices there are two main types of application that can be run on these devices:

- Native applications: the type of application can easily be interfaced with the mobile devices hardware (camera, accelerometer, GPS, ...)

- Web App: they have limited access to some native features of the mobile devices such as orientation, geolocalisation, media,...

Thus by the use of native applications instead of web app in telemedicine applications, the access to the mobile devices will be very efficient. Thus, logically, the qualities of the features associated with video, image and voice will be improved. Therefore the main health actors of teleexpertise activities will have better quality of information and then be able to make more informed decisions.

\section{Conclusion}

Telemedicine represents an important field of interest that is growing increasingly with the use of new technologies. Teleexpertise one of its acts in prison permits to reduce the cost of medical professionals movement to the prison or the transportation on inmates to hospitals.

The proposed solution shows a teleexpertise system which permits to medical professional to collaborate easily and efficiently using both mobile devices and PCs.

By the usage of the SOAP protocol in the architecture, the solution will permit to several communicating devices to interoperate thanks to a good level of structured information specification in Web Services format. Our model reaches four of the Telemedicine requirements specified in Mutafungwa et al. (2012). In other words, on the basis of the defined information framework, the proposed model checks coverage, QoS guarantees and prioritisation, mobility and roaming and service usability.

In future work we will focus on the semantic interoperability issue and argumentative reasoning for explaining medical decisions. We will develop the stage of formal ontology modelling to make explicit some semantic interoperability difficulties that are identified, providing a common framework for a semantically interoperable environment. Likewise, the semantic interoperability of medical information would enrich collaborative telemedicine projects, pragmatic knowledge organisation and the management of medical emergencies. In telemedicine practices (as with any medical practice) the activities are restricted by some legal and ethical constraints. Therefore, the efficient management of medical files and records is critical for any medical practice so that relevant information and patterns can be quickly retrieved. The argumentative reasoning formalises the elements of arguments underlying the decision-making processes as well as addressing the specific operational requirements that take into account the facts of contextual cases.

\section{References}

Adebesin, Funmi, Foster, Rosemary, KotzT, Paula, Van Greunen, Darelle, 2013. A review of interoperability standards in e-health and imperatives for their adoption in africa. South African Comput. J. 50, 55-72.

Altran, 2010. ICT \& health: Beyond technological innovation?

Barjis, J. et al., 2013. A sustainable and affordable support system for rural healthcare delivery, decision support systems. Elsevier, 2013. http://dx.doi.org/ 10.1016/j.dss.2013.06.005.

Batistatos, M.C., Tsoulos, G.V., Athanasiadou, G.E., 2012. Mobiletelemedicine for moving vehicle scenarios: Wireless technology options and challenges. J. Network Comput. Appl. 35, 1140-1150.

DGOS, 2012. Technical Working Group/Information Systems, French Ministry of Employment, Labour and Health. Recommendations for the implementation of a telemedicine project, Technical Deployment: Urbanization and infrastructure, March 2012.

Figueredo, M.V.M., Dias, J.S., 2004. Mobile telemedicine system for home care and patient monitoring. In: Proceedings of the 26th Annual International Conference of the IEEE EMBS, September 2004.

[On-line] Available: http://www.fipa.org/specs/fipa00029/SC00029H.pdf. Foundation for Intelligent Physical Agents (FIPA).

K. Hung and Y.T. Zhang. Implementation of a wap-based telemedicine system for patient monitoring. IEEE Transactions on Information Technology in Biomedicine, vol. 7(2), June 2003.

Iwayaa, L.H., Gomesa, M.A.L., Simplfcioa, M.A., Carvalhoa, T.C.M.B., Dominicinia, C.K., Sakuraguia, R.R.M., Rebelob, M.S., Gutierrezb, M.A., NSslundc, M., Hskanssonc, P., 2013. Mobile health in emerging countries: A survey of research initiatives in Brazil. Elsevier 82, $283-298$.

Knabe, Tore, Schillo, Michael, Fisher, Klaus. Improvements to the FIPA Contract Net Protocol for Performance Increase and Cascading Applications, July 2002. Kulkarni, Prajakta, Ozturk, Yusuf, 2011. mPHASIS: Mobile patient healthcare and sensor information system. J. Network Comput. Appl. 34, 402-417. 
Lai, Chien-Chih, Lee, Ren-Guey, Hsiao, Chun-Chieh, Liu, Hsin-Sheng, Chen, Chun-Chang, 2009. A h-qos-demand personalized home physiological monitoring system over a wireless multi-hop relay network for mobile home healthcare applications. J. Network Comput. Appl. 32, $1229-1241$.

Liekna, Aleksis, Lavendelis, Egons, Grabovskis, Arvids. Experimental Analysis of Contract NET Protocol in Multi-Robot Task Allocation. Applied Computer Systems. Riga Technical University. 13(1), 2012, 6-14. DOI: 10.2478/v10312-012-0001-7.

Mutafungwa, Edward, Zheng, Zhong, HSmSISinen, Jyri, Husso, Mika, Laitila, Matti, 2012. Femtocells for public safety communications: The emergency telemedicine case study. In: Saeed, Rashid A., Chaudhari, Bharat S., Mokhtar, Rania A. (Eds.), Femtocell communications and technologies: Business opportunities and deployment challengesa, pp. 241-270. DOI: 10.4018/978-1-4666-0092-8.ch012.

Simple Object Access Protocol. [On-line] Available: http://www.w3.org/2002/07/soap-translation/soap12-part0.html.

Unified modeling language (uml). [On-line] Available: http://www.uml.org/.

Extensible markup language (xml). [On-line] Available: http://www.w3.org/XML/. 